\title{
Effective Perihelion Advance and Potentials in a Conformastatic Background with Magnetic Field
}

\author{
Abraão J. S. Capistrano ${ }^{1,2}$ and Antonio C. Gutiérrez-Piñeres ${ }^{3,4}$ \\ ${ }^{1}$ Federal University for Latin American Integration, Apartado Postal 2123, 85867-970 Foz do Iguaçu, PR, Brazil \\ ${ }^{2}$ Casimiro Montenegro Filho Astronomy Center, Itaipu Technological Park, 85867-970 Foz do Iguaçu, PR, Brazil \\ ${ }^{3}$ Facultad de Ciencias Básicas, Universidad Tecnológica de Bolívar, CP 131001, Cartagena, Colombia \\ ${ }^{4}$ Instituto de Ciencias Nucleares, Universidad Nacional Autónoma de México, Apartado Postal 70543, 04510 Mexico City, Mexico
}

Correspondence should be addressed to Abraão J. S. Capistrano; capistranoaj@unb.br

Received 8 August 2016; Accepted 18 October 2016

Academic Editor: Josep M. Trigo-Rodríguez

Copyright (C) 2016 A. J. S. Capistrano and A. C. Gutiérrez-Piñeres. This is an open access article distributed under the Creative Commons Attribution License, which permits unrestricted use, distribution, and reproduction in any medium, provided the original work is properly cited.

Exact solutions of the Einstein-Maxwell field equations for a conformastatic metric with magnetized sources are investigated. In this context, effective potentials are studied in order to understand the dynamics of the magnetic field in galaxies. We derive the equations of motion for neutral and charged particles in a spacetime background characterized by this class of solutions. In this particular case, we investigate the main physical properties of the equatorial circular orbits and related effective potentials. In addition, we obtain an effective analytic expression for the perihelion advance of test particles. Our theoretical predictions are compared with the observational data calibrated with the ephemerides of the planets of the solar system and the Moon (EPM2011). In general, we show that the magnetic punctual mass predicts values that are in better agreement with observations than the values predicted in Einstein's gravity alone.

\section{Introduction}

Magnetics fields are extensively studied in literature and their influence on the galactic dynamics are currently subject of active research, for example, on the understanding of the galactic jets and inner process of "active" galaxy core, neutron stars dynamics [1], and/or movement of charged particles in spacetimes [2-4] or neutral particles in charged galactic halo [5-7]. In summary, they are present in almost every celestial object from stars, pulsars, and nearby galaxies to clusters of galaxies. An interesting review can be found in [812]. To this matter, the Einstein-Maxwell equations have been revealed to be an important tool to deal with this problem and help us on the understanding of the dynamics of magnetic fields in galaxies. Important approaches are the relativistic models with disk like configurations and relativistic disk accretion models as proposed in recent years [13-18] and references therein. In a recent publication [15], we studied the behavior of a test particle submitted to a magnetic field in a relativistic galaxy disk model and how its influence may affect the dynamics. In a different approach, in this paper we investigate effective potentials using Einstein-Maxwell equations motivated by the necessity to understand how the dynamics of a galaxy respond to flattening and how the magnetic field may be related to this process. This may be a basis for futures advances in both galactic and stellar formations. We also explore the possibility of an influence of magnetic field in the apsidal precession in solar system scale, in other words, how it might affect the movement of test particles embedded in solar gravitational field. To this matter, we use data calibrated with the ephemerides of the planets of the solar system and the Moon (EPM2011) [19, 20].

The present paper is divided into sections. In Section 2, we study the basic framework of a conformastatic background and investigate some applications using the isothermalsphere logarithm potential and Toomre-Kuzmin-like potential, which are compatible with axisymmetric systems. In Section 3, we obtain an expression for the perihelion advance of a charged test particle in a generic conformastatic spacetime 
in the presence of a magnetic field and perform a comparison between our results, the results from Einstein's gravity alone, and the values observed for the secular perihelion precession of some inner planets and minor objects of the solar system. In the conclusion section, we make the final considerations.

\section{The Conformastatic Background}

General relativistic scenaries described by using a conformally flat space of orbits are very alluring from mathematical point of view and in physical applications. Using the definition proposed by Synge [21], we start our analysis with the use of conformally flat space, which is the main characteristic of a conformastatic spacetime (e.g., the Schwarzschild metric). Considering the background of a conformastatic gravitational source in presence of a magnetic field described by the line element in standard cylindrical coordinates, one can write [14]

$$
d S^{2}=-e^{2 \phi} d t^{2}+e^{-2 \phi}\left(d r^{2}+d z^{2}+r^{2} d \varphi^{2}\right),
$$

where the metric potential $\phi$ depends only on the variables $r$ and $z$. The vacuum Einstein-Maxwell equations in geometrized units, such that $c=8 \pi G=\mu_{0}=\epsilon_{0}=1$, are given by

$$
\begin{aligned}
G_{\alpha, \beta} & =E_{\alpha \beta}, \\
F_{; \beta}^{\alpha \beta} & =0,
\end{aligned}
$$

where $F_{\alpha \beta}=A_{\beta, \alpha}-A_{\alpha, \beta}$ and $E_{\alpha \beta}$ is the electromagnetic energy-momentum tensor.

$$
E_{\alpha \beta}=\frac{1}{4 \pi}\left\{F_{\alpha \gamma} F_{\beta}^{\gamma}-\frac{1}{4} g_{\alpha \beta} F_{\gamma \delta} F^{\gamma \delta}\right\} .
$$

The Greek indices run from 1 to 4 .

With the electromagnetic potential $A_{\alpha}=\left(0,0,0, A_{\varphi}(r\right.$, $z)$ ) and the line element in (1) the Einstein-Maxwell equations in (2a) and (2b) are equivalent to the system of equations

$$
\begin{aligned}
\nabla \cdot\left(r^{-2} e^{2 \phi} \nabla A_{\varphi}\right) & =0, \\
\nabla^{2} \phi-\nabla \phi \cdot \nabla \phi & =0, \\
\phi_{r}^{2}-\frac{1}{2 r^{2}} e^{2 \phi} A_{\varphi, z}^{2} & =0, \\
\phi_{z}^{2}-\frac{1}{2 r^{2}} e^{2 \phi} A_{\varphi, r}^{2} & =0, \\
\phi_{r} \phi_{z}+\frac{1}{2 r^{2}} e^{2 \phi} A_{\varphi, r} A_{\varphi, z} & =0 .
\end{aligned}
$$

By using the procedure to obtain solutions of the EinsteinMaxwell equations presented in [14], suitable solutions of the system in (4a), (4b), (4c), (4d), and (4e) can be displayed as

$$
\begin{aligned}
e^{\phi} & =\frac{1}{1-U}, \\
A_{\varphi, r} & =\sqrt{2} r U_{, z}, \\
A_{\varphi, z} & =-\sqrt{2} r U_{, r},
\end{aligned}
$$

where $U(r, z)$ is a solution of Laplace's equation.
2.1. Motion of Test Charged Particles. The motion of a test particle of charge $q$ and mass $m$ moving in a magnetized background is described by the Lagrangian

$$
\mathscr{L}=\frac{1}{2} m u_{\alpha} u^{\alpha}+q A_{\alpha} u^{\alpha}
$$

where $u^{\mu}=d x^{\mu} / d s, s$ being an arbitrary parameter. The corresponding Hamiltonian of the particle is

$$
\mathscr{H}=\frac{1}{2 m}\left(p^{\mu}-q A^{\mu}\right)\left(p_{\mu}-q A_{\mu}\right)
$$

where the canonical momentum is given by $p_{\mu}=m u_{\mu}+q A_{\mu}$. The motion equations are given by

$$
\begin{aligned}
u^{\mu} & =\frac{\partial \mathscr{H} g^{\mu \nu}}{\partial p^{\nu}}, \\
\frac{d p^{\mu}}{d s} & =-\frac{\partial \mathscr{H} g^{\mu \nu}}{\partial p^{\nu}},
\end{aligned}
$$

where $\mathscr{H}_{c} \equiv p_{\mu} p^{\mu} /(2 m)$. Accordingly, by introducing (7) into (8a) and (8b) we obtain

$$
\begin{aligned}
& \frac{d p^{t}}{d s}=0, \\
& \frac{d p^{\varphi}}{d s}=0, \\
& \frac{d p^{r}}{d s}=\frac{p^{\mu} p_{\mu}}{2 m} \frac{\partial g^{r r}}{\partial r}, \\
& \frac{d p^{z}}{d s}=\frac{p^{\mu} p_{\mu}}{2 m} \frac{\partial g^{z z}}{\partial z} .
\end{aligned}
$$

From (7) and the normalization condition $u^{\mu} u_{\mu}=-\varepsilon$ (with $\varepsilon=(1,0,-1)$ for space-like, null, and time-like curves) we have the condition

$$
\mathscr{H}=-\frac{1}{2} m \varepsilon .
$$

On the another hand, from (9a) and (9b), we have

$$
p^{t}=\text { constant } \equiv-E
$$

and also

$$
p^{\varphi}=\text { constant } \equiv L,
$$

respectively, whereas, from (9c) and (9d), we obtain

$$
\begin{aligned}
& \ddot{r}=W \frac{\partial g^{r r}}{\partial r}, \\
& \ddot{z}=W \frac{\partial g^{r r}}{\partial r},
\end{aligned}
$$

where

$$
W \equiv \frac{1}{2}\left(\varepsilon+\frac{q^{2}}{m^{2}} A_{\varphi} A^{\varphi}-\frac{2 q L}{m^{2}} A_{\varphi}\right) .
$$


We can write the last system in the form

$$
\begin{aligned}
& \ddot{r}=-\frac{\partial \Phi_{\mathrm{eff}}}{\partial r}, \\
& \ddot{z}=-\frac{\partial \Phi_{\mathrm{eff}}}{\partial r},
\end{aligned}
$$

where

$$
d \Phi_{\mathrm{eff}}=W \frac{\partial g^{r r}}{\partial r} d r+W \frac{\partial g^{r r}}{\partial z} d z .
$$

$\Phi_{\text {eff }}$ is called the "effective potential" (see equations (3.68) pg. 160 in [16]). In terms of the solution in (5a), (5b), and (5c) one obtains

$$
\begin{aligned}
& \ddot{r}=-\frac{\partial \Phi_{\mathrm{eff}}(U)}{\partial r}, \\
& \ddot{z}=-\frac{\partial \Phi_{\mathrm{eff}}(U)}{\partial z},
\end{aligned}
$$

where

$$
\begin{aligned}
d \Phi_{\mathrm{eff}}(U)= & -\frac{h(r, z)}{(1-U)^{3}} d U, \\
h(r, z) \equiv & \varepsilon+\frac{2}{m^{2} r^{2}(1-U)^{2}}\left(\frac{\partial}{\partial z} \int_{0}^{r} U r d r\right)^{2} \\
& -\frac{2 \sqrt{2} q L}{m^{2}} \frac{\partial}{\partial z} \int_{0}^{r} U r d r .
\end{aligned}
$$

Thus the three-dimensional motion of the particle in an axissymmetric potential can be reduced to the two-dimensional motion of the particle in a "Newtonian potential" $U(r, z)$.

2.2. Circular Motion in the Plane $z=0$. To study the circular motion of the test charged particle we start with the conditions

$$
\begin{aligned}
\dot{r} & =0, \\
\frac{\partial \Phi_{\mathrm{eff}}}{\partial r} & =0 .
\end{aligned}
$$

Then, from the first of these equations, (7) and (10), we have the energy of the particle as follows:

$$
E^{2}=-g^{t t}\left(\varepsilon m^{2}+g_{\varphi \varphi}\left(L-q A_{\varphi} g^{\varphi \varphi}\right)^{2}\right) .
$$

From the second condition in (20) we have

$$
\ddot{r}=W \frac{\partial g^{r r}}{\partial r}=-\frac{\partial \Phi_{\mathrm{eff}}}{\partial r}=0 .
$$

Notice that if $W=0$, from (21) and (14), we obtain

$$
E^{2}=\frac{g_{\varphi \varphi}}{g_{t t}} L^{2}
$$

Thus, by introducing the corresponding metric coefficients of the line element in (1), such as

$$
E^{2}=-r^{2} e^{-4 \phi} L^{2}
$$

which lacks physical meaning, hence the condition $\partial \Phi_{\text {eff }} /$ $\partial r=0$ is equivalent to

$$
\begin{gathered}
\frac{\partial g^{r r}}{\partial r}=0, \\
W \neq 0 .
\end{gathered}
$$

The minimum radius for stable circular orbit occurs in the inflection points of the effective potential. Thus we must solve the equation

$$
\ddot{r}=\frac{\partial^{2} \Phi_{\mathrm{eff}}}{\partial r^{2}}=0
$$

or, equivalently, solve the equation

$$
\frac{\partial^{2} g^{r r}}{\partial r^{2}}=0
$$

On the other hand, by calculating the derivative with respect to $z$ in both sides of (21), we obtain for the angular moment

$$
L=q A_{\varphi} g^{\varphi \varphi}+\frac{l}{\left(g^{t t} g_{\varphi \varphi}\right)_{, z}},
$$

where

$$
\begin{aligned}
l \equiv & q A_{\varphi} g^{t t} g_{\varphi \varphi} g^{\varphi \varphi}{ }_{, z} \\
& \pm \sqrt{\left(q A_{\varphi} g^{t t} g_{\varphi \varphi} g^{\varphi \varphi}{ }_{, z}\right)^{2}-\varepsilon m^{2} g_{, z}^{t t}\left(g^{t t} g_{\varphi \varphi}\right)_{, z}},
\end{aligned}
$$

and we have used the Einstein-Maxwell equation $\phi_{r}^{2}=(1 /$ $\left.2 r^{2}\right) e^{2 \phi} A_{\varphi, z}^{2}$. By substituting this value for $L$ in (21) we obtain the energy of the particle as follows:

$$
E^{2}=-g^{t t}\left(\varepsilon m^{2}+g_{\varphi \varphi} \frac{l^{2}}{\left(g^{t t} g_{\varphi \varphi}\right)_{, z}^{2}}\right) .
$$

Since the Lagrangian in (6) does not depend explicitly on the variables $t$ and $\varphi$, one can obtain the following two conserved quantities:

$$
p_{t}=-m c e^{2 \phi} \dot{t} \equiv-\frac{E}{c}
$$

and also

$$
p_{\varphi}=m r^{2} e^{-2 \phi} \dot{\varphi}+\frac{q}{c} A_{\varphi} \equiv L,
$$

where $E$ and $L$ are, respectively, the energy and the angular momentum of the particle as measured by an observer at rest at infinity. Furthermore, the momentum $p_{\alpha}$ of the particle can be normalized so that $g_{\alpha \beta} \dot{x}^{\alpha} \dot{x}^{\beta}=-\Sigma$. Accordingly, for the metric in (1), we have

$$
-e^{2 \phi} \dot{t}^{2}+e^{-2 \phi}\left(\dot{r}^{2}+\dot{z}^{2}+r^{2} \dot{\varphi}^{2}\right)=-\Sigma,
$$

where, with $c=1$, the notation $\Sigma=1,0,-1$ denotes spacelike, null, and time-like curves, respectively. 
As an application of (18), we use an axial bidimensional isothermal potential, which has the form

$$
U(r)=1-v_{0}^{2} \ln \left(r^{2}+z^{2}\right),
$$

and straightforwardly we get the expression

$$
\begin{aligned}
d \Phi_{\mathrm{eff}}= & \frac{4}{v_{0}^{4}}\left\{\epsilon+a\left(\frac{z}{r}\right)^{2}+b z \ln \left(z^{2}+r^{2}\right)\right\} \\
& \cdot \frac{r d r+z d z}{\left(z^{2}+r^{2}\right) \ln \left(z^{2}+r^{2}\right)^{3}} .
\end{aligned}
$$

Hence, integrating the former expression, it is necessary to obtain a convergence of the integral away from origin; we use a Laurent expansion $\sum_{k=1}^{\infty}=1 / k^{2} \sim \pi^{2} / 6$. Finally, after long algebra, we can write the form of the effective potential felt by charged particle with mass moving with velocity $v_{0}$ and total angular momentum $L$ as follows:

$$
\begin{aligned}
\Phi_{\mathrm{eff}}(r, z)= & \frac{\epsilon}{4 v_{0}^{4}}\left[\frac{\ln ^{2}\left(z^{2}+r^{2}\right)-4 \ln ^{2} z}{\ln ^{2} z \ln ^{2}\left(z^{2}+r^{2}\right)}\right] \\
& +\frac{b z}{v_{0}^{4}}\left[\frac{\ln \left(z^{2}+r^{2}\right)-2 \ln z}{\ln z \ln \left(z^{2}+r^{2}\right)}\right] \\
& -\frac{a z^{2}}{v_{0}^{4} r^{2} \ln ^{2}\left(z^{2}+r^{2}\right)} \\
& +\frac{a z^{2} \pi^{2}}{24 v_{0}^{4} \ln ^{2}\left(z^{2}+r^{2}\right)}
\end{aligned}
$$

where we denote $a=2 v_{0}^{2} / m^{2}$ and $b=\left(2 \sqrt{2} / m^{2}\right) q L v_{0}^{2}$. In Figure 1, we notice that a small value of the velocity $v_{0}$ induces outgoing lines from the center as expected, as noted in the three panels. In (b), we notice that time-like curves suggest that the magnetic lines distort the path of a test charged particle away from the center of the galaxy.

In the same sense, we investigate a Toomre-Kuzmin-like potential since we are dealing with an axisymmetric system, which has the form

$$
U(r)=1-\frac{\alpha}{\sqrt{\left(r^{2}+z^{2}\right)}},
$$

where $\alpha$ is a unitary free parameter to guarantee the correct units, and, straightforwardly, one can get the expression

$$
\begin{aligned}
& d \Phi_{\mathrm{eff}}=\frac{2 r}{\alpha^{2}}\left\{\epsilon+\frac{2}{\alpha m^{2} r^{2}}\left(z-\sqrt{\left(r^{2}+z^{2}\right)}\right)^{2}\right. \\
& \left.+\frac{2 \sqrt{(2)} q \alpha L}{m}\left(\frac{z-\sqrt{\left(r^{2}+z^{2}\right)}}{\sqrt{\left(r^{2}+z^{2}\right)}}\right)\right\}\left(r^{2}+z^{2}\right)(r d r \\
& +z d z) .
\end{aligned}
$$

And, after a long algebra, we can write the form of the effective potential felt by charged particle with mass moving with total angular momentum $L$ as follows:

$$
\begin{aligned}
\Phi_{\mathrm{eff}}(r, z)= & P(z) r^{3}+U r^{5}-K(z) \ln |z| \\
& +V(r, z) \sqrt{\left(r^{2}+z^{2}\right)}+I(r, z) \\
& +C(z) \ln \left|r+\sqrt{\left(r^{2}+z^{2}\right)}\right|
\end{aligned}
$$

where we denote the following terms:

$$
\begin{aligned}
P(z)= & \frac{2 \epsilon}{3 \alpha^{2}} z^{2}+\frac{4}{m^{2} \alpha^{3}} z^{2}+\frac{3 \epsilon}{2 \alpha^{2}}+\frac{3}{m^{2} \alpha^{3}} \\
& -\frac{3 \sqrt{(2)}}{m \alpha} q L-\frac{4 \sqrt{(2)}}{3 m \alpha} q L z^{2}, \\
U= & \frac{1}{5}\left(\frac{2 \epsilon}{\alpha^{2}}+\frac{4}{m^{2} \alpha^{3}}\right)-\frac{4}{5} \frac{\sqrt{(2)}}{m \alpha} q L, \\
K(z)= & \frac{3}{m^{2} \alpha^{3}} z^{5}+\frac{\sqrt{(2)}}{2 m \alpha} q L z^{3}, \\
V(r, z)= & \frac{4 r z}{m^{2} \alpha^{3}}\left(r^{2}+z^{2}\right)+\frac{3}{m^{2} \alpha^{3}} z^{3} \\
& +\frac{\sqrt{(2)}}{2} \frac{q L}{m \alpha} r z\left(2 r^{2}+z^{2}\right), \\
I(r, z)= & \frac{8 r}{\alpha^{3} m^{2}} z^{4}, \\
C(z)= & \frac{3}{m^{2} \alpha^{3}} z^{5}-\frac{\sqrt{(2)}}{2} \frac{q L}{m \alpha} z^{3} .
\end{aligned}
$$

In Figure 2, we do not have any considerable difference between the three figures and around the origin it is possible to check the singularity and the lines away to the center.

On the other hand, we can express the effective potential directly related to energy. In doing so, we use the relations in (31), (32), and (33) that give three linear differential equations, involving the four unknowns $\dot{x}^{\alpha}$. It is possible to study the motion of test particles with only these relations, if we limit ourselves to the particular case of equatorial trajectories; that is, $z=0$. Indeed, since the gravitational configuration is symmetric with respect to the equatorial plane, a particle with initial state $z=0$ and $\dot{z}=0$ will remain confined to the equatorial plane which is, therefore, a geodesic plane. Substituting the conserved quantities of (31) and (32) into (33), we find

$$
\dot{r}^{2}+\Phi_{\mathrm{eff}}=\frac{E^{2}}{m^{2} c^{2}}
$$

where

$$
\Phi_{\mathrm{eff}}(r) \equiv \frac{L^{2}}{m^{2} r^{2}}\left(1-\frac{q A_{\varphi}}{L c}\right)^{2} e^{4 \phi}+\Sigma e^{2 \phi}
$$

is an effective potential. We assume the convention that the positive value of the energy corresponds to the positivity of 


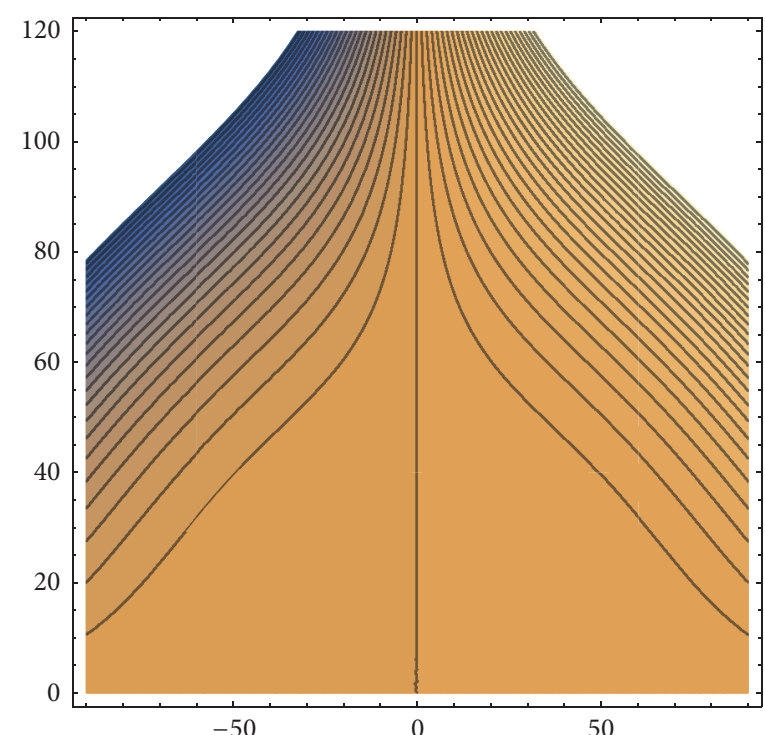

(a)

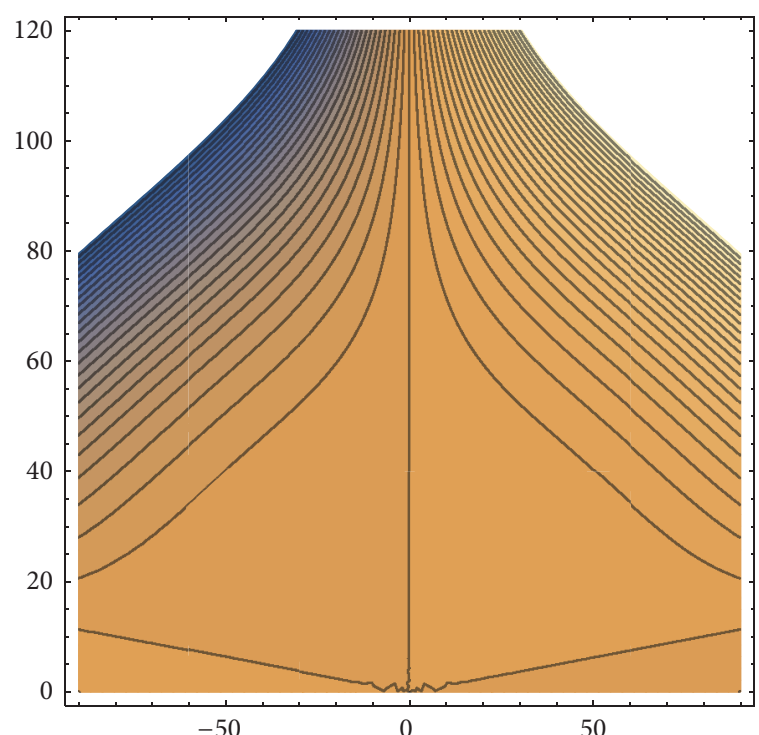

(b)

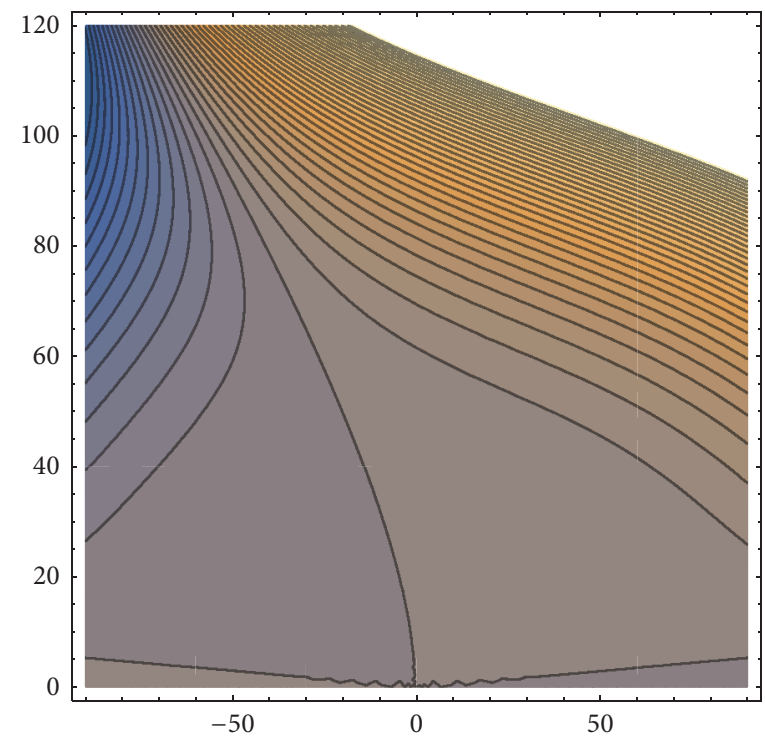

(c)

FIGURE 1: The figures are made, respectively, with $(\epsilon=1,0,-1)$ with fixed parameter $v_{0}=0.02$ and unitary value for mass and charge with 50 contour lines in the ranges $r[-90,90]$ and $z[0,120]$.

the solution $E_{ \pm}= \pm m c \Phi_{\text {eff }}^{1 / 2}$. Consequently, $E_{+}=-E_{-}=$ $m c \Phi_{\text {eff }}^{1 / 2}$.

The motion of charged test particles is governed by the behavior of the effective potential in (42). The radius of circular orbits and the corresponding values of the energy $E$ and angular momentum $L$ are given by the extrema of the function $\Phi_{\text {eff. }}$. Therefore, the conditions for the occurrence of circular orbits are

$$
\begin{aligned}
\frac{d \Phi_{\mathrm{eff}}}{d r} & =0, \\
\Phi_{\mathrm{eff}} & =\frac{E^{2}}{m^{2} c^{2}} .
\end{aligned}
$$

Thus, by calculating the condition in (43) for the effective potential in (42), we find the angular momentum of the particle in circular motion

$$
\begin{aligned}
L_{c \pm}= & \frac{q A_{\varphi}}{c} \\
& +\frac{q r A_{\varphi, r} e^{\phi} \pm \sqrt{\left(q r A_{\varphi, r} e^{\phi}\right)^{2}-4 \Sigma c^{2} m^{2} r^{3} \phi_{, r}\left(2 r \phi_{, r}-1\right)}}{2 c e^{\phi}\left(2 r \phi_{, r}-1\right)} .
\end{aligned}
$$

Conventionally, we can associate the plus and minus signs in the subscript of the notation $L_{c \pm}$ to dextrorotation and levorotation, respectively. Moreover, by inserting the value of the angular momentum in (44) into the second equation of (43), 

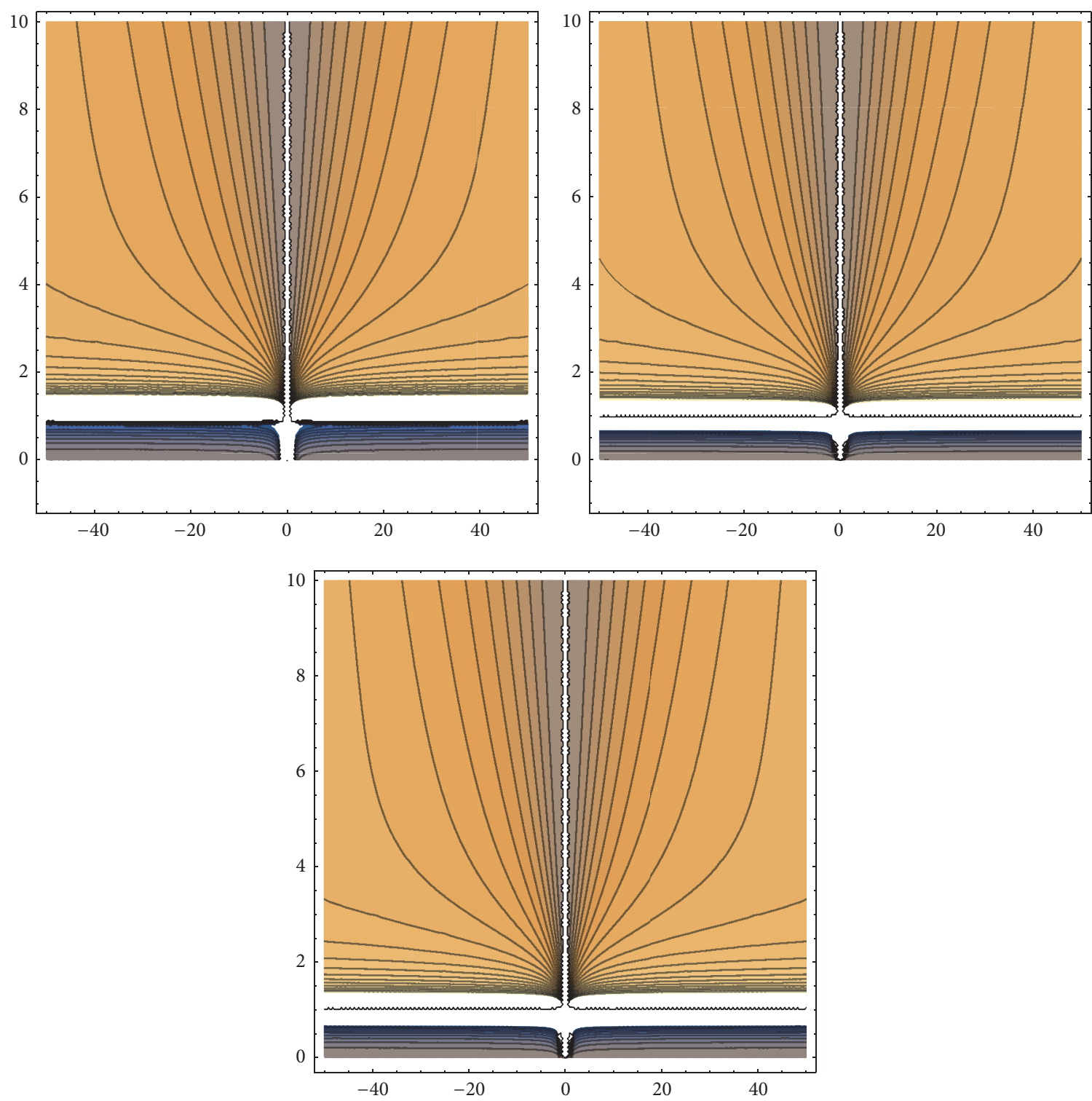

Figure 2: The figures are made, respectively, with $(\epsilon=1,0,-1)$ with fixed parameters $m=1, q=1, L=1$, and $\alpha=1$, for 30 contour lines in ranges $r[-50,50]$ and $z[-1,10]$.

we obtain the energy $E_{c \pm}^{( \pm)}$of the particle in a circular orbit as

$$
E_{c \pm}^{( \pm)}= \pm m c e^{\phi}\left(\Sigma+\xi_{c}^{( \pm)}\right)^{1 / 2}
$$

where

$$
\begin{aligned}
& \xi_{c}^{( \pm)} \\
& =\frac{\left[q r A_{\varphi, r} e^{\phi} \pm \sqrt{\left(q r A_{\varphi, r} e^{\phi}\right)^{2}-4 \Sigma c^{2} m^{2} r^{3} \phi_{, r}\left(2 r \phi_{, r}-1\right)}\right]^{2}}{4 m^{2} c^{2} r^{2}\left(2 r \phi_{, r}-1\right)^{2}} .
\end{aligned}
$$

Therefore, each sign of the value of the energy corresponds to two kinds of motions (dextrorotation and levorotation) indicated in (45) and (46) by the superscripts $( \pm)$.

\section{Perihelion Advance in a Conformastatic Magnetized Spacetime}

One of the most important tests of general relativity and modified theories of gravitation in astrophysical scale is the perihelion advance of celestial objects. In this section, we present the analytic expressions, which determine the perihelion advance of charged test particle moving in a conformastatic spacetime under the presence of a magnetic field. Starting with the first integral in (33), we restrict the 
analysis to the motion of a particle on the plane with $z=0$, since in solar scales the planets, comets, and asteroids revolve essentially on the plane of the orbits. Then, we have

$$
\begin{aligned}
& \left(\frac{d r}{d \varphi}\right)^{2}=-r^{2}\left[1+\frac{m^{2} r^{2}}{\left(L-(q / c) A_{\varphi}\right)^{2}}\left(\Sigma(1-U)^{2}\right.\right. \\
& \left.\left.-\frac{E^{2}}{m^{2} c^{2}}(1-U)^{4}\right)\right]
\end{aligned}
$$

where all the quantities are evaluated at $z=0$ and we have used the expressions for the energy and angular momentum of the particle given by (31) and (32), respectively. With the change of variable $u=1 / r,(47)$ can be transformed into

$$
\frac{d^{2} u}{d \varphi^{2}}+u^{2}=F(u)
$$

where

$$
F(u) \equiv \frac{1}{2} \frac{d G}{d u}
$$

$$
\begin{aligned}
G(u) & \equiv \frac{1}{\left(1-q A_{\varphi} / c L\right)^{2}}\left[\frac{E^{2}}{c^{2} L^{2}}(1-U)^{4}\right. \\
& \left.-\frac{\Sigma m^{2}}{L^{2}}(1-U)^{2}\right] .
\end{aligned}
$$

Accordingly, by following the procedure proposed in [25], we have for the resulting perihelion advance

$$
\delta \varphi=\pi\left(\frac{d F}{d u}\right)_{u=u_{0}}
$$

where $u_{0}$ is the radius of a nearly circular orbit, which is given by the roots of the equation $F\left(u_{0}\right)=u_{0}$. In (51), we have shown the procedure to obtain an expression for the perihelion advance of a charged test particle in a generic conformastatic spacetime with a magnetic field. We now illustrate the results by considering a particular conformastatic spacetime generated from the harmonic potential of a punctual mass

$$
\begin{aligned}
U(r, z) & =-\frac{G M}{c^{2} R}, \\
R^{2} & =r^{2}+z^{2} .
\end{aligned}
$$

Thus, by inserting (52) into (49), we obtain for $F(u)$

$$
F(u)=\frac{\left[\left(2 E^{2} G M / c^{4} L^{2}\right)\left(1+\left(G M / c^{2}\right) u\right)^{3}-\left(\Sigma m^{2} G M / c^{2} L^{2}\right)\left(1+\left(G M / c^{2}\right) u\right)\right]}{(1-q \sqrt{G} M / c L)^{2}} .
$$

Accordingly, the perihelion advance of a particle in this spacetime is given by

$$
\delta \varphi=\pi \frac{\left[\left(6 E^{2} G^{2} M^{2} / c^{6} L^{2}\right) x_{0}^{2}-\Sigma m^{2} G^{2} M^{2} / c^{4} L^{2}\right]}{(1-q \sqrt{G} M / c L)^{2}}
$$

where the term

$$
x_{0} \equiv 1+\frac{G M}{c^{2}} u_{0}
$$

satisfies the equation

$$
\begin{aligned}
& 2 E^{2} G^{2} M^{2} x_{0}^{3} \\
& -\left[\sum m^{2} G^{2} M^{2} c^{2}+c^{6} L^{2}\left(1-\frac{q \sqrt{G} M}{c L}\right)^{2}\right] x_{0} \\
& +c^{6} L^{2}\left(1-\frac{q \sqrt{G} M}{c L}\right)^{2}=0 .
\end{aligned}
$$

Thus, by inserting the real solution of (56) into (53), we find that the perihelion advance of the test particle orbit is given by

$$
\delta \varphi=\pi \frac{\psi_{0}-k_{2}^{2}}{Q^{2}}
$$

where

$$
\psi_{0} \equiv \frac{\left[6\left(Q^{2}+k_{2}^{2}\right)+\left[54 Q^{2} k_{1}\left(-1+\sqrt{1-6\left(Q^{2}+k_{2}^{2}\right)^{3} / 81 Q^{4} k_{1}^{2}}\right)\right]^{2 / 3}\right]^{2}}{6\left[54 Q^{2} k_{1}\left(-1+\sqrt{1-6\left(Q^{2}+k_{2}^{2}\right)^{3} / 81 Q^{4} k_{1}^{2}}\right)\right]^{2 / 3}},
$$


with

$$
\begin{aligned}
& k_{1}^{2}=\frac{E^{2} G^{2} M^{2}}{c^{6} L^{2}}, \\
& k_{2}^{2}=\frac{\sum m^{2} G^{2} M^{2}}{c^{4} L^{2}}
\end{aligned}
$$

and also

$$
Q^{2}=\left(1-\frac{q \sqrt{G} M}{c L}\right)^{2} .
$$

Notice that when $q=0$ (and, consequently, $Q=1$ ) we get the case in which (54) describes the perihelion advance of a neutral particle. Actually, we restrict ourselves to the neutral case, since objects like planets, asteroids, and comets are neutral on average and the consideration of a charge is hardly significant. Moreover, this neutrality is essentially due to the influence of the solar wind, but a global net charge, for example, in stars, is still on discussion [26].

In order to get a real use of (57), we follow the procedure presented in [25]. First, we rewrite both the angular momentum (44) and the energy (45), which depend on the radial distance $r$ in terms of the parameters that describe the orbit of rotating test particles. For the radial distance, one can use the ellipse formula in the Euclidean plane as

$$
r=\frac{s\left(1-\epsilon^{2}\right)}{1+\epsilon \cos \varphi},
$$

where $s$ is the semimajor axis and $\epsilon$ the eccentricity of the orbit. Moreover, we can rewrite (57) by using physical units related to observations as

$$
\delta \varphi^{\star}=\pi \gamma^{\star} \frac{\left(\psi_{0}-k_{2}^{2}\right) s^{2}}{Q^{2} M_{\odot} T^{2}},
$$

where we have introduced the solar mass $M_{\odot}$ and the period $T$ of the rotating body. The parameter $\gamma^{\star}=((180 / \pi) / 3600) T$ allows us to transform units from radians to (secular) degrees. Moreover, in order to obtain a real effective advance $\delta \varphi_{\text {eff }}$ and to alleviate the error propagation, we define a deviation formulae away from general relativity standard result $\delta \varphi_{\text {eins }}$ induced by the coupled Einstein-Maxwell fields as

$$
\delta \varphi_{\mathrm{eff}}=\delta \varphi_{\mathrm{eins}} \pm \beta_{0} \delta \varphi^{\star},
$$

where a dimensionless parameter $\beta_{0}$ measures the small variation of the orbits through time. As we have checked in the studied cases in Table 1 , a variation of $\beta_{0}$ must not exceed $10 \%$ of the ratio between the Einstein-Maxwell contribution $\delta \varphi^{\star}$ and observations $\delta \varphi_{\text {obs }}$.

When applied to the observational data [23], plus supplementary precession corrections from EPM2011 [19, 20], one can test (63). Thus, we obtain the results presented in Table 1 for the perihelion precession of inner planets of the solar system, two NEO's asteroids named 433 Eros and 3200
Phaethon and NEO 2p/Encke comet. The data for the astrophysics parameters of planets, like semimajor axis, eccentricity, period, and mass, can be found in JPL solar system dynamics (http://ssd.jpl.nasa.gov/?planets) and for asteroids and comets, in JPL small body database (http://ssd.jpl.nasa.gov/ sbdb.cgi). The orbital periods are in units of years.

As shown in Table 1, the theoretical results match the observations, and a slight improvement is obtained as compared to the standard Einstein gravity which turns our model closer to the observations. We conclude that the gravitational interaction generated by the magnetic field of the central body can play an important role in astrophysical observations. It is worth saying that the values of $\alpha$ seem to be sensitive to the variation of the eccentricity of the orbits and the mass of the object as seen in the studied cases and the values have a close resemblance to PPN parameters that have a bound $|2 \gamma-\beta-1|<3 \times 10^{-3}$ [27].

In addition, some other considerations must be noted. The constant $\Sigma$ enters explicitly the expression for the perihelion advance in (33), and it represents null, time-like, and space-like curves. For $\Sigma=0$, we do not have a solution since (63) diverges. For time-like trajectories, $\Sigma=-c^{2}$ no physical results are obtained, because in the corresponding Newtonian limit a differential equation is obtained, whose solution implies that $r$ is negative. Moreover, no significant differences were found for different values of the charge of the order $q / m \sim 10^{-3}$, which is the value where the behavior of the energy and angular momentum becomes affected by the presence of the effective charge. In the same sense, no differences could be found when using both solutions for the angular momentum $L_{c \pm}$ and energy $E_{c \pm}$.

\section{Conclusion}

In this work we have shortly shown the characteristics of the motion of a charged particle along circular orbits in spacetime described by a conformastatic solution of the EinsteinMaxwell equations. As a particular example we have considered the case of a charged particle moving in the gravitational field of a punctual source placed at the origin of coordinates. Our analysis is based on the study of the behavior of an effective potential that determines the position and stability properties of circular orbits. We also have investigated the behavior of effective potentials. Interestingly, we have noticed time-like curves in a particular axial bidimensional isothermal potential, where the effective magnetic field does exert influence on the movement of a particle. On the other hand, a larger sample of effective potentials must be studied in order to verify if this pattern occurs more frequently, which can be fundamental to understand how galaxies form and evolve.

In addition, we have also calculated an expression for the perihelion advance of a test particle in general magnetized conformastatic spacetime obtaining a good agreement with the observed values for the perihelion of inner solar planets and some selected NEO asteroids. It is worth noting that all results presented were obtained with the initial assumption of a neutral particle, in accordance with the fact that planets are largely neutral. Specifically, in the perihelion drift, we find that the differences between a neutral particle and a charged 
TABLE 1: Comparison between the values of secular precession of inner planets in units of arcsec/century $\left(" \cdot \mathrm{cy}^{-1}\right)$ of the standard $($ Einstein) perihelion precession $\delta \varphi_{\text {eins }}$ [22] for neutral test particles (planets, asteroids/comets) in the conformastatic magnetized spacetime of a punctual mass $\delta \phi_{\text {eff }}$. The data for $\delta \varphi_{\text {obs }}$ stands for the secular observed perihelion precession in units of arcsec/century adapted from [23] by adding a supplementary precession correction from EPM2011 [19, 20]. In addition, the results for the NEOS 433 Eros, 3200 Phaethon, and 2p/Encke comet are also presented. The mass of the $2 \mathrm{p} /$ Encle comet as $m=3.85 \times 10^{13} \mathrm{~kg}$ was estimated with a bulk density $\rho=0.5 \mathrm{~g} \cdot \mathrm{cm}^{3}$ as shown in $[24]$.

\begin{tabular}{lcccc}
\hline Object & $\delta \varphi_{\text {obs }}$ & $\delta \varphi_{\text {eins }}$ & $\delta \varphi_{\text {eff }}$ & $\beta_{0}$ \\
\hline Mercury & $43.098 \pm 0.503$ & 42.97817 & 42.9782 & $0.7605 \times 10^{-4}$ \\
Venus & $8.026 \pm 5.016$ & 8.62409 & 8.62425 & $0.1426 \times 10^{-2}$ \\
Earth & $5.00019 \pm 1.00038$ & 3.83848 & 3.83944 & $0.4375 \times 10^{-2}$ \\
Mars & $1.36238 \pm 0.000537$ & 1.35086 & 1.36980 & $0.3729 \times 10^{-1}$ \\
433 Eros & 1.60 & 1.57317 & 1.58668 & $0.2906 \times 10^{-1}$ \\
3200 Phaethon & 10.1 & 10.1201 & 10.1213 & $0.3499 \times 10^{-2}$ \\
2p/Encke & 1.9079 & 1.868 & 1.92833 & $0.5623 \times 10^{-1}$ \\
\hline
\end{tabular}

particle are slightly small, when realistic values for the effective charge are used. This means that the electromagnetic interaction between the charge and the central magnetized body does not seriously affect the value of the perihelion advance. Nevertheless, the magnetic field enters explicitly the metric components and, consequently, affects the motion of neutral test particles through the gravitational interaction. This explains why the numerical predictions of the perihelion advance generated by a punctual magnetized mass are in better agreement with observations than the predictions of Einstein's theory alone. As a future prospect, we will apply the Poincaré surface-of-section method for analyzing weakly perturbed Hamiltonian conformastatic systems.

\section{Competing Interests}

The authors declare that there are no competing interests regarding the publication of this paper.

\section{References}

[1] M. Bocquet, S. Bonazzola, E. Gourgoullon, and J. Novak, "Rotating neutron star models with a magnetic field," Astronomy \& Astrophysics, vol. 301, 1995.

[2] E. Hackman and H. Xu, "Charged particle motion in Kerr-Newmann space-times," Physical Review D, vol. 87, no. 12, Article ID 124030, 21 pages, 2013

[3] K. Chakraborty, F. Rahaman, S. Ray, A. Nandi, and N. Islam, "Possible features of galactic halo with electric field and observational constraints," General Relativity and Gravitation, vol. 46, no. 10, article 1807, pp. 1-24, 2014.

[4] A. Nandi and N. Islam, "Possible features of galactic halo with electric field and observational constraints," General Relativity and Gravitation, vol. 46, article 1807, 2014.

[5] D. Pugliese, H. Quevedo, and R. Ruffini, "Circular motion of neutral test particles in Reissner-Nordström spacetime," Physical Review D, vol. 83, no. 2, Article ID 024021, 2011.

[6] D. Pugliese, H. Quevedo, and R. Rffini, "Motion of charged test particles in Reissner-Nordström spacetime," Physical Review D, vol. 83, no. 10, Article ID 104052, 2011.
[7] D. Pugliese, H. Quevedo, and R. Ruffini, "Equatorial circular motion in Kerr spacetime," Physical Review D, vol. 84, no. 4, Article ID 044030, 2011.

[8] J. Han, "The large-scale magnetic field structure of our Galaxy: efficiently deduced from pulsar rotation measures," in Proceedings of the Magnetized Interstellar Medium Symposium, B. Uyanker, W. Reich, and R. Wielebinski, Eds., vol. 24, pp. 3-12, Antalya, Turkey, 2003.

[9] J. Han, "Magnetic fields in our milky way galaxy and nearby galaxies, solar and astrophysical dynamos and magnetic activity," in Proceedings of the International Astronomical Union, IAU Symposium, Solar and Astrophysical Dynamos and Magnetic Activity, A. G. Kosovichev, E. M. de Gouveia Dal Pino, and Y. Yan, Eds., vol. 294, pp. 213-224, 2012.

[10] M. Krause, "Magnetic fields in spiral galaxies," in The Magnetized Interstellar Medium, B. Uyanker, W. Reich, and R. Wielebinski, Eds., 2003.

[11] R. Beek and R. Wielebinski, Planets, Stars and Stellar Systems, vol. 614, Springer, 2013.

[12] R. Beck, "Magnetic fields in spiral galaxies," The Astronomy and Astrophysics Review, vol. 24, no. 1, article 4, 2016.

[13] D. Voigt and P. S. Letelier, "Exact relativistic static charged perfect fluid disks," Physical Review D, vol. 70, no. 6, Article ID 0664003, 2004.

[14] A. C. Gutiérrez-Piñeres, G. A. González, and H. Quevedo, "Conformastatic disk-haloes in Einstein-Maxwell gravity," Physical Review D, vol. 87, no. 4, Article ID 044010, 2013.

[15] A. C. Gutiérrez-Piñeres, A. J. S. Capistrano, and H. Quevedo, "Test particles in a magnetized conformastatic spacetime," Physical Review D, vol. 93, no. 12, Article ID 124009, 2016.

[16] J. Binney and S. Tremaine, Galactic Dynamic, Princeton University Press, Princeton, NJ, USA, 2011.

[17] A. C. Gutiérrez-Piñeres and A. J. S. Capistrano, "Exact relativistic magnetized haloes around rotating disks," Advances in Mathematical Physics, vol. 2015, Article ID 916026, 13 pages, 2015.

[18] A. J. S. Capistrano, W. L. Roque, and R. S. Valada, "Weyl conformastatic perihelion advance," Monthly Notices of the Royal Astronomical Society, vol. 444, no. 2, pp. 1639-1646, 2014.

[19] N. P. Pitjev and E. V. Pitjeva, "Constraints on dark matter in the solar system," Astronomy Letters, vol. 39, no. 3, pp. 141-149, 2013. 
[20] E. V. Pitjeva and N. P. Pitjev, "Relativistic effects and dark matter in the solar system from observations of planets and spacecraft," Monthly Notices of the Royal Astronomical Society, vol. 432, no. 4, pp. 3431-3437, 2013.

[21] J. Synge, Relativity: The General Theory, North-Holland Pub. Co., Interscience Publishers, Amsterdam, The Netherlands, 1960.

[22] K. Wilhelm and B. N. Dwivedi, "Secular perihelion advances of the inner planets and asteroid Icarus," New Astronomy, vol. 31, pp. 51-55, 2014.

[23] G. G. Nambuya, "Azimuthally symmetric theory of gravitation - I. On the perihelion precession of planetary orbits," Monthly Notices of the Royal Astronomical Society, vol. 403, no. 3, pp. 1381-1391, 2010.

[24] J. A. Fernandez, Comets: Nature, Dynamics, Origin, and their Cosmogonical Relevance, vol. 328 of Astrophysics and Space Science Library, Springer, Berlin, Germany, 2005.

[25] T. Harko, Z. Kovács, F. S. N. Lobo, and R. Soc, "Solar System tests of Hořava-Lifshitz gravity," Proceedings of the Royal Society of London A: Mathematical, Physical and Engineering Sciences, vol. 467, no. 2129, pp. 1390-1407, 2011.

[26] L. Neslušan, "On the global electrostatic charge of stars," Astronomy \& Astrophysics, vol. 372, no. 3, pp. 913-915, 2001.

[27] C. M. Will, "The confrontation between general relativity and experiment," Living Reviews in Relativity, vol. 9, article 3, 2006. 

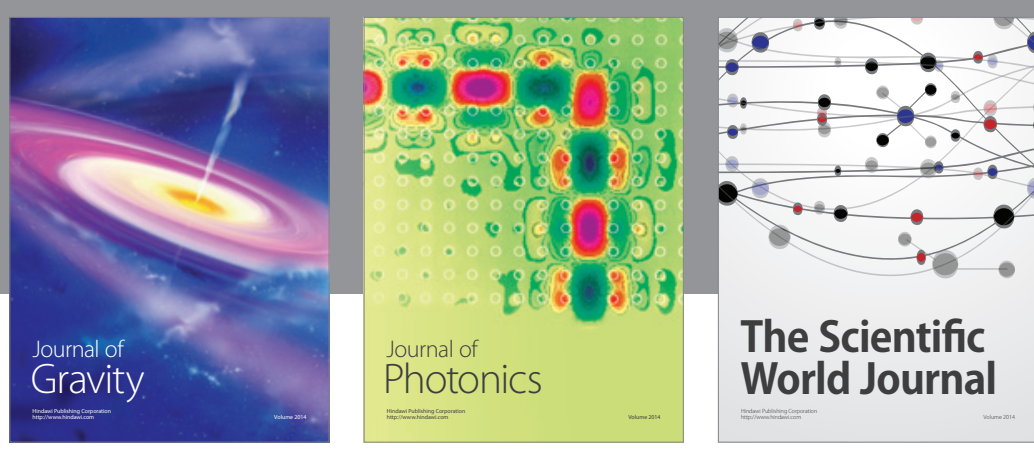

The Scientific World Journal
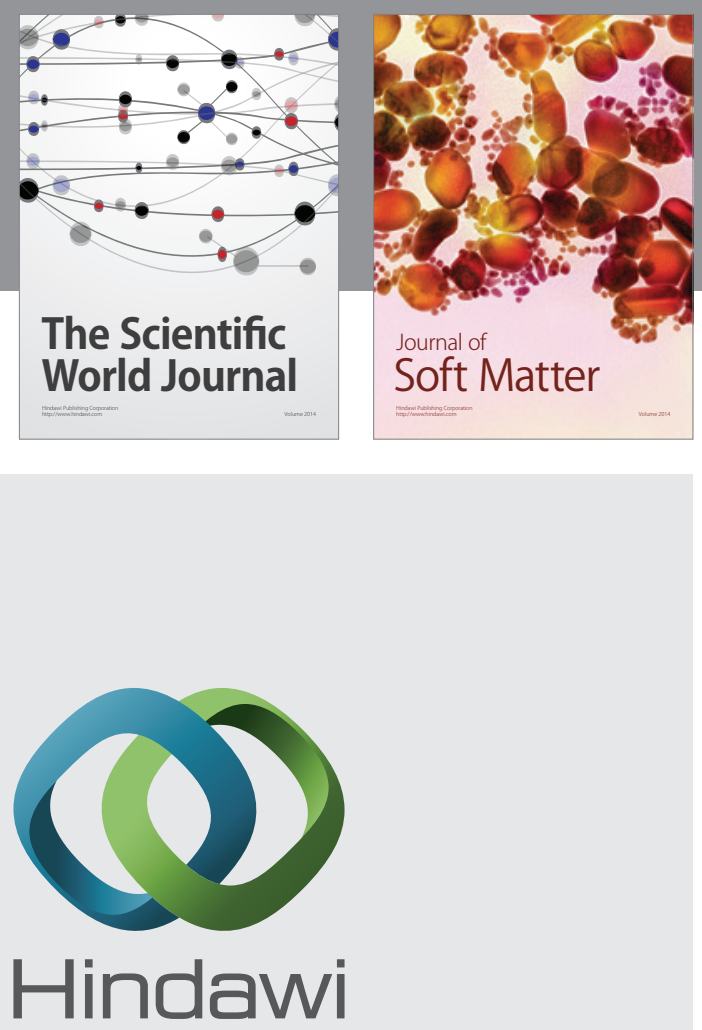

Submit your manuscripts at

http://www.hindawi.com

nternational Journal of

Statistical Mechanics
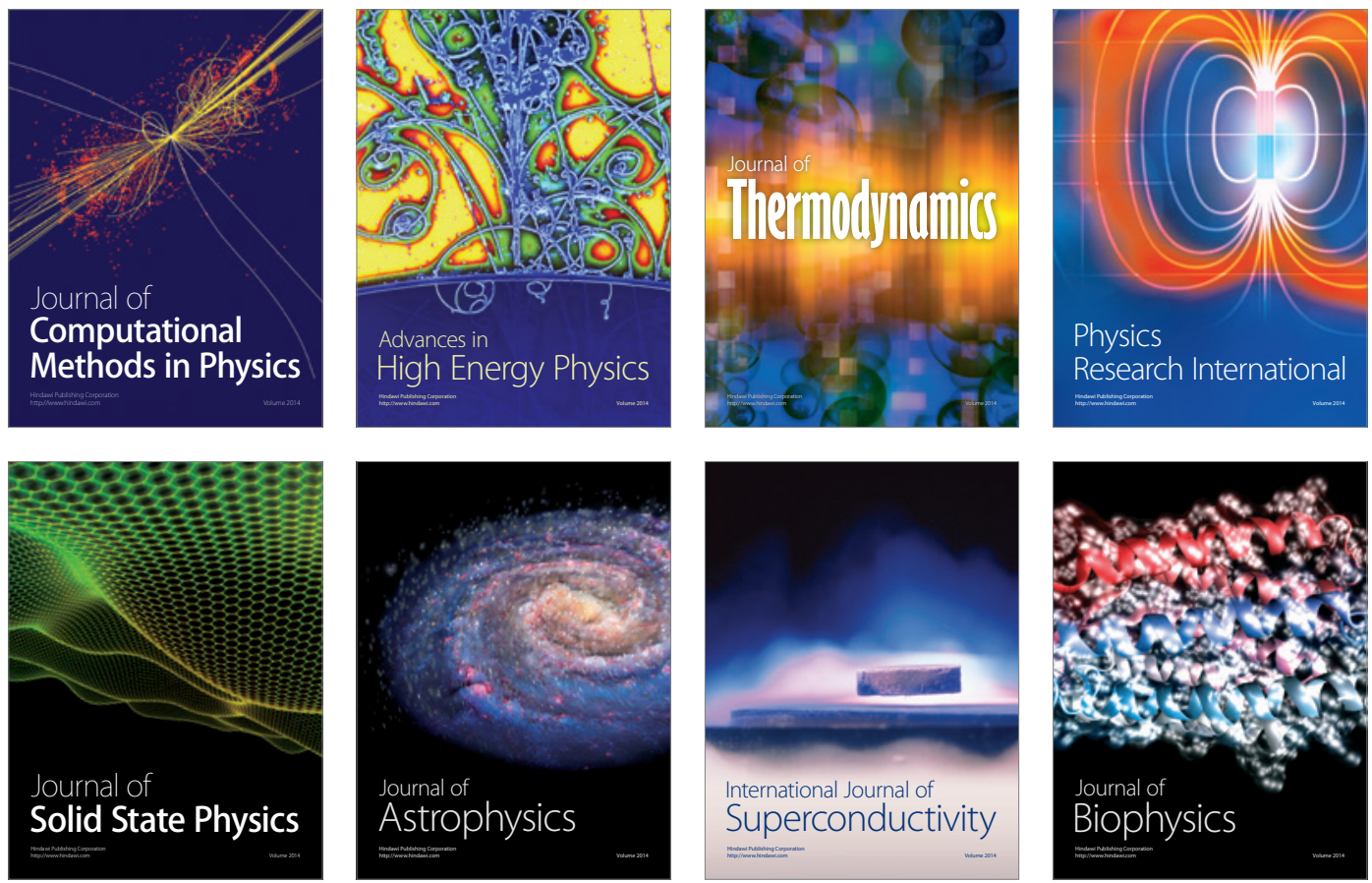
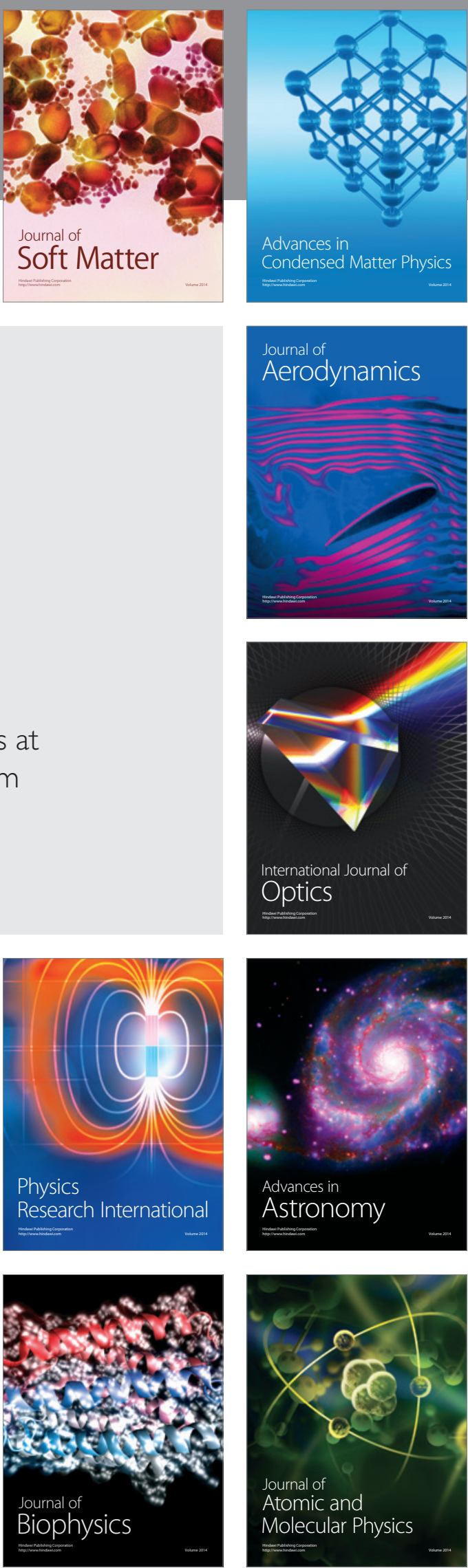